\title{
Role of testosterone and photoperiod on seasonal changes in horn growth and sperm variables in the Iberian ibex: a model for polygynous wild bovids
}

\author{
J Santiago-Moreno, A Gómez-Brunet, A Toledano-Díaz, R Salas-Vega ${ }^{1}$, F Gómez-Guillamón ${ }^{1}$ and \\ A López-Sebastián \\ Department of Animal Reproduction, INIA, Avenida Puerta de Hierro Km 5.9, Madrid 28040, Spain \\ ${ }^{1}$ Consejería de Medio Ambiente, Junta de Andalucía, Málaga 29006, Spain \\ (Correspondence should be addressed to J Santiago-Moreno; Email: moreno@inia.es)
}

\begin{abstract}
This work examines the effect of testosterone secretion and photoperiod on seasonal changes in horn growth and sperm variables in the Iberian ibex (Capra pyrenaica), here used as a model for polygynous wild bovids. The hypothesis that high levels of testosterone provide an endocrine signal that inhibits horn growth in autumn was tested by assessing the effect of cyproterone acetate (CA), an anti-androgen, administered in October - coinciding with the period of natural increases in plasma testosterone concentrations - under different photoperiodic conditions (natural photoperiod and artificial long days). The persistence of horn growth during autumn in all ibexes held under the long-day photoperiodic conditions clearly shows that horn growth regulation in the mating season is primarily modulated by day length and not by a fall in testosterone concentration. A retrospectively designed
\end{abstract}

second experiment involving testosterone propionate (TP) administration in April (when horns are growing) was then undertaken to confirm that high levels of testosterone do not inhibit horn growth. Overall, the results strongly suggest that the rise in testosterone secretion during the autumn mating season does not act as an endocrine signal for the arrest of horn growth, although the rate of horn growth before the mating season may be related to springtime testosterone levels. A direct relationship was seen between the rate of horn growth and the incidence of sperm abnormalities. Neither CA treatment in October nor TP administration in April affected the studied sperm variables. By contrast, CA treatment plus artificial long days in autumn had a negative effect on sperm motility and sperm morphology.

Journal of Endocrinology (2012) 214, 155-163

\section{Introduction}

Horns are appendages used by the males of polygynous bovid species to compete for females during the mating season. In addition to their function in combat, these secondary sexual characteristics appear to provide a sensitive indicator of genetic stress (Parsons 1992) and to serve as signals of male vigour that females may use to select mates (Geist 1966, 1991). Certainly, it has been shown that wild bovid males with the largest and most symmetrical horns - those that are usually dominant - produce the best quality sperm (Roldan et al. 1998, Santiago-Moreno et al. 2007). The reproductive success of bovid males is therefore related to pre-copulatory strategies such as combat ability, which is strongly related to horn development, as well as to post-copulatory strategies such as sperm competition (Preston et al. 2003).

In most wild ruminant species living at temperate or higher latitudes, the photoperiod is the main environmental cue regulating seasonal breeding activity (Hafez 1952). The annual arrest of horn growth in wild bovids coincides with the time when males are producing their highest levels of testosterone, i.e. in the autumn, when the photoperiod is shortening and when spermatogenesis is at its height (Lincoln 1990, 1998, Coloma et al. 2011). When spermatogenesis decreases in spring, horn growth begins again (Fowler 1993). It has been suggested that this seasonal pattern may be modulated by testosterone secretion (Lincoln 1998, Santiago-Moreno et al. 2005a). Adequate horn growth in spring has a high energy demand, and it may be that the high levels of testosterone seen in the mating season provide an endocrine signal that stops this growth, thus allowing energy use to be refocused towards combat and spermatogenesis. Certainly, spermatogenesis is known to be modulated by this hormone (Courot \& Ortavant 1981). To test this idea, testosterone secretion was manipulated at the end of normal horn growth in the Iberian ibex (Capra pyrenaica), a polygynous wild bovid that shows strongly seasonal sexual activity and horn development (Toledano-Díaz et al. 2007). If androgens are required to stop horn growth during the mating season, antiandrogen treatment should prolong the horn growth period. 
The annual cycle of horn growth in these animals is regulated directly by photoperiod, although the mechanism is unclear (Toledano-Díaz et al. 2007). Thus, an anti-androgen treatment was administered under different photoperiodic conditions. A second experiment was then designed to confirm the negative hypothesis arising from the results, i.e. that high testosterone levels have no influence on the end of horn growth. Given the relationship between seasonal changes in horn development and sperm quality, the influence of artificial photoperiod and testosterone manipulation on testis size and a number of sperm variables was also analysed.

\section{Materials and Methods}

Cyproterone acetate (CA; Androcur, Schering A.G., Berlin, Germany), a progestational androgen receptor blocker, was prepared for injecting into the test animals according to the method of Jaczewski et al. (2004) with minor modifications. CA (3.2 g) was dissolved in $7 \mathrm{ml}$ benzyl benzoate, and this was then combined with $25 \mathrm{ml}$ of olive oil (final CA concentration $100 \mathrm{mg} / \mathrm{ml}$ ). The final mixture was heated for $10 \mathrm{~min}$ at $120{ }^{\circ} \mathrm{C}$ with agitation to encourage appropriate dissolution.

Testosterone propionate (TP; Fluka, Sigma-Aldrich) was prepared for injecting into the test animals by dissolving $25 \mathrm{mg}$ of TP in $2 \mathrm{ml}$ of olive oil. The mixture was gently agitated before administration.

Diluents for sperm collection were prepared in the laboratory using reagent-grade chemicals purchased from Panreac Química S.A. (Barcelona, Spain) and Sigma Chemical Co.

\section{Animals}

Twenty-five adult Iberian ibex males (body weight: $48-57 \mathrm{~kg}$ ) were housed in captivity at the INIA Department of Animal Reproduction (Madrid, $40^{\circ} 25^{\prime} \mathrm{N}$ ). This flock consists of animals obtained from the Sierra de CazorlaSegura and Serranía de Ronda Game Reserves in Southern Spain, plus others born at the INIA facilities. The age of the animals (4-6 years) was calculated by counting the horn rings (Fandos 1995). To alleviate stress during the experimental procedures, animals were accustomed over a 1-year period before the experiment to handling in a small restraining stall $\left(2 \mathrm{~m}^{2}\right)$ in which blood samples were collected and horn and testicular measurements taken. During all manipulations, the eyes were covered with a mask to further reduce stress. All animals were fed with Visan K59 (Visan Ind. Zoot., Madrid, Spain), which provides a balanced diet, supplemented with barley grain, barley straw, and dry alfalfa. Water, mineral, and vitamin blocks were made freely available. All handling procedures were approved by the INIA Ethics Committee and were performed in accordance with the Spanish Policy for Animal Protection RD1201/2005, which conforms to the European Union Directive 86/609 regarding the protection of animals used in scientific experiments.

\section{Experimental procedure}

Experiment 1: influence of anti-androgen CA treatment in autumn under different photoperiodic conditions This experiment lasted from February to December 2008; blood samples for testosterone analysis and horn and scrotal circumference measurements were taken from the beginning (to determine horn growth in February, measurements were obviously taken in January as well).

Ibexes were randomly distributed into four groups:

1) Control group $(\mathrm{PHPn}+\mathrm{oil})$ composed of four ibexes kept under natural photoperiodic conditions (natural variations in day length from $15 \mathrm{~h}$ light/day at the summer solstice to $9 \mathrm{~h} /$ day at the winter solstice) and given $2 \mathrm{ml}$ i.m. olive oil without CA twice weekly (Tuesday and Friday) from 30 September to 28 October.

2) Long days +olive oil group (PHPld+oil) composed of four ibexes kept in an open stable exposed to long days of $15 \mathrm{~h}$ light:9h darkness (equivalent to the summer solstice photoperiod) for 6 months between 21 June and 21 December. This photoperiod was regulated using an electric clock that operated fluorescent tubes providing an artificial light intensity of $\sim 350$ lux at floor level. Animals from this group were given $2 \mathrm{ml}$ i.m. olive oil without CA twice weekly (Tuesday and Friday) from 30 September to 28 October.

3) Long days + CA group (PHPld + CA) composed of four ibexes kept in an open stable exposed to artificial long days (15 h light:9 h darkness) between 21 June and 21 December, and given $200 \mathrm{mg}$ i.m. CA twice weekly (Tuesday and Friday) from 30 September to 28 October, coinciding with the period of natural rises in plasma testosterone concentrations (Toledano-Díaz et al. 2007). This protocol has been successfully used at our laboratory to maintain plasma testosterone concentrations at basal levels.

4) Natural photoperiod $+C A$ group $(\mathrm{PHPn}+\mathrm{CA})$ composed of four ibexes kept under natural photoperiodic conditions and given $200 \mathrm{mg}$ CA i.m. twice weekly (Tuesday and Friday) from 30 September to 28 October.

Experiment 2: influence of testosterone administration in spring As the results of Experiment 1 did not support the initial hypothesis (i.e. that the increase in autumnal testosterone inhibits horn growth), this second experiment was designed to confirm that a rise in plasma testosterone concentration does not act as an endocrinal signal for the inhibition of horn growth. The experiment lasted from January to September 2010 with all measurement made as in Experiment 1. 
Ibexes were randomly distributed into the following two groups:

1) Control group $(\mathrm{PHPn}+\mathrm{oil})$ composed of four ibexes kept under natural photoperiodic conditions and given $2 \mathrm{ml}$ olive oil without TP s.c. every $48 \mathrm{~h}$ from 27 March to 28 April.

2) Testosterone-treated group ( $\mathrm{PHPn}+\mathrm{TP})$ composed of five ibexes kept under natural photoperiodic conditions and administered $25 \mathrm{mg}$ TP s.c. in $2 \mathrm{ml}$ olive oil every $48 \mathrm{~h}$ from 27 March to 28 April, coinciding with the natural increase in horn growth (Toledano-Díaz et al. 2007).

\section{Collection of samples and measurements}

Horn and scrotal circumference measurements were taken twice monthly (2 weeks apart) over the entire experimental period. Blood samples were normally taken twice monthly before and after CA or TP injection, although collection was daily in the first week after injection, and twice weekly for the remainder of the first month following injection. Each time, three blood samples (1000, 1100, and $1200 \mathrm{~h}$ ) were collected from the jugular vein into heparinised tubes $(4 \mathrm{ml})$. The collected blood was centrifuged at $1500 \mathrm{~g}$ for $15 \mathrm{~min}$. The plasma was separated and a pool of $1 \mathrm{ml}$ aliquots of the three samples was made and stored at $-20{ }^{\circ} \mathrm{C}$ until required for testosterone concentration analysis. The rate of horn growth was measured with a measuring tape, covering the distance from the base of the horn to a reference mark cut into the upper outside edge of the horn. Horn measurements were taken for both horns. The circumference of the scrotum was measured at its widest diameter using a scrotal measuring tape (Ideal Instruments, Neogen Corporation, Schiller Park, IL, USA).

Semen was recovered by electroejaculation as previously described (Santiago-Moreno et al. 2009) using a Lane Pulsator IIIZ electroejaculator (Lane Manufacturing, Inc., Denver, CO, USA). Sperm was recovered from each animal of each group at least once every month, from October to December in Experiment 1 and from April to June in Experiment 2.

\section{Hormone analyses}

Testosterone concentrations were measured by RIA in duplicate plasma aliquots $(100 \mu \mathrm{l})$ as described previously (Santiago-Moreno et al. 2005b). All samples were analysed in a single assay. The sensitivity was $0.05 \mathrm{ng} / \mathrm{ml}$. The intra-assay coefficient of variation (CV) was $11 \%(n=7)$.

\section{Semen evaluation}

Ejaculate volumes were measured using a micropipette (Gilson, Villiers Le Bel, France). Total sperm concentration was determined using a Neubauer chamber (Marienfeld, Lauda-Königshofen, Germany). Motility was assessed after $30 \mathrm{~min}$ incubation at $37^{\circ} \mathrm{C}$. The percentage of motile spermatozoa and the quality of motility were evaluated subjectively via phase-contrast microscope (Zeiss, Oberkochen, Germany) observations made at $100 \times$. The vigour of sperm movement was scored on a 0 (lowest) to 5 (highest) scale. Plasma membrane integrity was assessed using the hypo-osmotic swelling test (Jeyendran et al. 1984) and by staining an aliquot of sperm suspension with nigrosin-eosin (Campbell et al. 1956). The percentage of spermatozoa with intact acrosomes was assessed in samples fixed in buffered $2 \%$ glutaraldehyde solution at $37^{\circ} \mathrm{C}$, using phase-contrast microscopy (magnification $1000 \times$; Pursel \& Johnson 1974). Individual spermatozoa that showed a smooth, crescentshaped apical ridge were classified as having an intact acrosome. Spermatozoa classified as not showing acrosome integrity were those with an irregularly shaped apical ridge, an absent apical ridge, or a loose and vesiculated acrosomal cap. Morphological abnormalities were assessed by phasecontrast microscopic examination of glutaraldehyde-fixed samples. Spermatozoa classified as showing abnormal morphology were those with abnormal head, a mid-piece defect, a cytoplasmic droplet, a coiled tail, or a bent tail. All analyses required the observation of 200 cells.

\section{Statistical analysis}

The plasma testosterone data, sperm volume, and sperm concentration showed a skewed distribution, as determined by the Shapiro-Wilk's test; values were therefore logtransformed before analysis. The remaining sperm variables (with values expressed as percentages) also showed a skewed distribution; their values were therefore arcsine transformed before analysis.

The effect of the interaction CA treatment $X$ photoperiod (first experiment) and the effect of TP (second experiment), on plasma testosterone concentrations, sperm variables, scrotal circumference, and horn development, were assessed using general linear model (GLM) repeated measures ANOVA. In the model, the photoperiodic condition (PHPn-PHPld) and treatment (oil-CA in Experiment 1, oil-TP in Experiment 2) were included as categorical predictor variables, age was included as a continuous predictor variable, and months (February-December) as within-subject factors (repeated measures). Differences between groups in different months were analysed by ANOVA. The arrest of horn growth was deemed to have begun in the first month to show a significant reduction in such growth ( $t$-test for matched pairs) compared with the three previous consecutive months with a high rate of horn growth. The resurgence of horn growth was taken as the first month showing a significant increase in horn growth ( $t$-test for matched pairs) compared with the previous consecutive months. Similar criteria were used to determine the onset of increasing scrotal circumference. Finally, variability in horn measurements between groups was compared via the mean CV. The possible link between horn growth and plasma testosterone concentration was examined by linear correlation analysis (Pearson). 


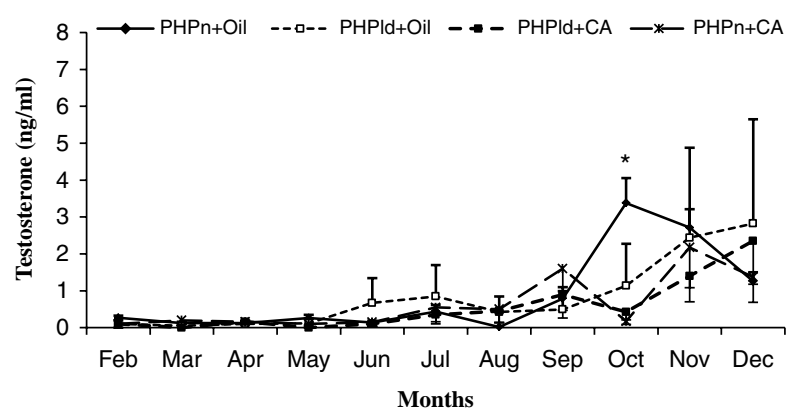

Figure 1 Changes in plasma testosterone concentration in the control (PHPn + oil group; filled diamond), $\mathrm{PHPld}+$ oil group (open square), $\mathrm{PHPld}+\mathrm{CA}$ (filled square), and $\mathrm{PHPn}+\mathrm{CA}(\times)$ groups. The time of CA administration in PHPn + CA and PHPId + CA groups was twice weekly from September 30th to October 28th. Asterisks indicate significant differences (ANOVA, $P<0.05$ ) between groups within months: the testosterone concentrations in the $\mathrm{PHPn}+$ oil group were higher than that in the PHPn $+\mathrm{CA}$ and PHPld $+\mathrm{CA}$ groups. PHPn, natural photoperiodic conditions; PHPId, artificial long-day conditions; CA, treated with cyproterone acetate in olive oil; oil, olive oil without cyproterone acetate.

Data are presented as means \pm S.E.M. Significance was set at $P<0 \cdot 05$. All calculations were performed using Statistica Software for Windows v.10.0, Series 2011 (StatSoft, Inc., Tulsa, OK, USA).

\section{Results}

Experiment 1: influence of anti-androgen CA treatment in autumn under different photoperiodic conditions

The PHPn+oil control group showed the characteristic seasonal pattern of testosterone secretion, with basal levels until September, and then a significant increase in its output. This was followed by a reduction in December (Fig. 1). This pattern was noticeably modified in the ibexes kept under the artificial photoperiod (Fig. 1), with a rapid decline in plasma testosterone concentration ( $t$-test for matched pairs, $P<0 \cdot 01)$ observed, coinciding with CA administration (October) in both the PHPn + CA and PHPld + CA animals. Scrotal circumference began to increase ( $t$-test for matched pairs, $P<0 \cdot 05)$ in September in the PHPn + oil and PHPn + CA groups, and in October in the PHPld+oil group (Fig. 2). The inhibitory effect of the long-day photoperiod on scrotal circumference first became evident in the PHPld + CA group (Fig. 2). GLM repeated measures ANOVA showed the interaction CA treatment $\times$ photoperiod to have no significant effect on horn growth. Neither did CA treatment have any independent effect on this growth. However, long days were seen to maintain horn growth during the autumn (GLM repeated measures ANOVA, $P<0 \cdot 01)$, independent of treatment with CA (Fig. 3). Horn growth was maintained in the PHPld + CA and PHPld + oil groups until the end of the experiment (Fig. 3), although in the PHPld + CA group it tended to decrease ( $t$-test for matched pairs, $P=0 \cdot 07)$ in December. By contrast, in both the PHPn+oil and PHPn + CA groups (Fig. 3), reduction ( $t$-test for matched pairs, $P<0.05)$ in horn growth occurred in November. Further, in both groups under artificial long days, monthly horn growth from June to December $(0 \cdot 9 \pm 0 \cdot 1$ and $0 \cdot 8$ $\pm 0.1 \mathrm{~cm}$ in the PHPld+CA and PHPld+oil groups respectively) was greater (ANOVA, $P<0.05$ ) than in the ibexes held under the natural photoperiod $(0 \cdot 5 \pm 0 \cdot 01$ and $0.5 \pm 0.04 \mathrm{~cm}$ in the PHPn+oil and PHPn + CA groups respectively). The CVs for the horn growth measurements in the PHPld + oil and PHPld + CA groups since June (the time of exposure to long days) were more homogeneous $(\mathrm{CV}=30 \cdot 2$ and $44.8 \%$ respectively) than in the $\mathrm{PHPn}+$ oil and $\mathrm{PHPn}+\mathrm{CA}$ groups (CV $=65.4$ and $50 \%$ respectively), in which the reduction in horn growth in autumn was not prevented.

No relationship was seen between plasma testosterone concentration and the moment at which horn growth reduction began. In the controls (PHPn + oil group; Fig. 1), the physiological increase in plasma testosterone concentrations in October was followed by a reduction in horn growth in November. The rapid decline in testosterone levels after CA administration in October under natural photoperiodic conditions (PHPn + CA group; Fig. 1) did not affect the reduction in horn growth (Fig. 3), which occurred at the same time as in the controls. The artificial long-day photoperiod (PHPld + CA and PHPld+oil groups) clearly altered the pattern of testosterone secretion (Fig. 1). This variable testosterone pattern was not associated with maintained horn growth in the autumn (Fig. 3). Indeed, no significant correlation was seen, in any experimental group, between horn growth and plasma testosterone concentration $(P>0 \cdot 05)$ from the time of CA treatment to the end of the experiment $\left(\mathrm{PHPn}+\right.$ oil, $R^{2}=0 \cdot 42 ; \mathrm{PHPn}+\mathrm{CA}, R^{2}=0 \cdot 02$; PHPld +CA, $R^{2}=0 \cdot 04$; and PHPld + oil, $\left.R^{2}=0 \cdot 05\right)$.

The interaction CA treatment $X$ photoperiod had a significant (GLM repeated measures ANOVA, $P<0 \cdot 05$ )

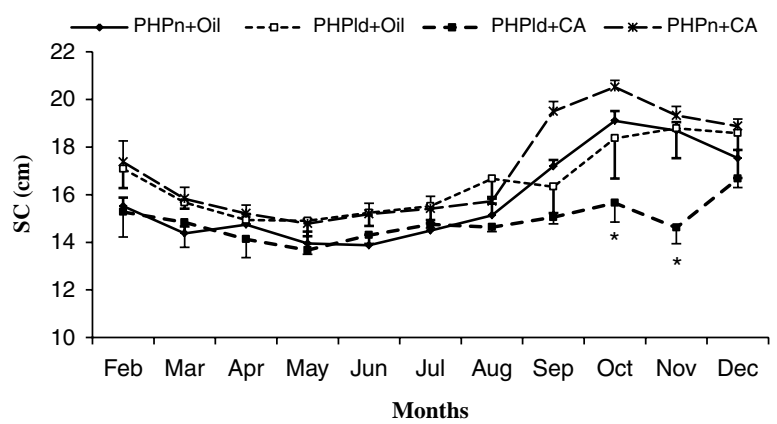

Figure 2 Seasonal changes in scrotal circumference (SC) in the control (PHPn + oil group; filled diamond), PHPld + oil group (open square), PHPld +CA (filled square), and $\mathrm{PHPn}+\mathrm{CA}(\times)$ groups. Asterisks indicate significant differences (ANOVA, $P<0 \cdot 05$ ) between groups within months: $\mathrm{SC}$ in the PHPId +CA group was lower than that in the remaining groups. PHPn, natural photoperiodic conditions; PHPId, artificial long-day conditions; CA, treated with cyproterone acetate in olive oil; oil, olive oil without cyproterone acetate. 


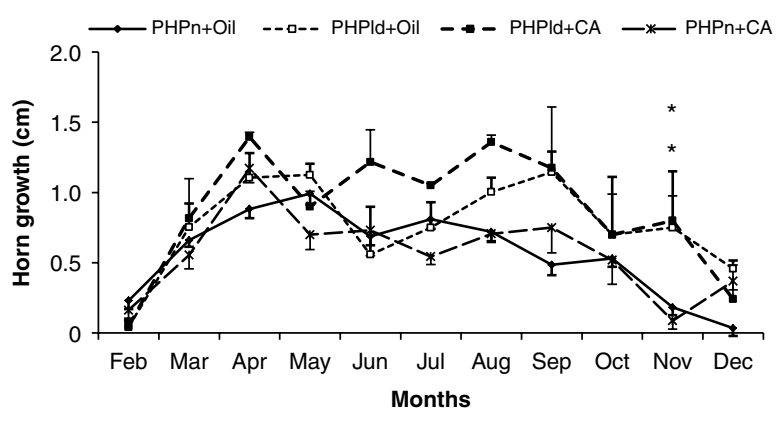

Figure 3 Seasonal changes in horn growth in the control $(\mathrm{PHPn}+$ oil group; filled diamond), PHPId + oil group (open square), PHPld + CA (filled square), and PHPn $+C A(X)$ groups. Asterisks indicate significant differences (ANOVA, $P<0 \cdot 05$ ) between groups within months: horn growth in the PHPId +CA and PHPId+oil groups was greater than that in the $\mathrm{PHPn}+$ oil and $\mathrm{PHPn}+\mathrm{CA}$ groups. PHPn, natural photoperiodic conditions; PHPId, artificial long-day conditions; $\mathrm{CA}$, treated with cyproterone acetate in olive oil; oil, olive oil without cyproterone acetate.

effect on the percentage of motile spermatozoa, with lower motility seen in the PHPld + CA group. A higher rate of sperm abnormalities (GLM repeated measures ANOVA, $P<0 \cdot 05)$ was seen in the PHPld+CA and PHPld+oil groups than in either PHPn group (Table 1).

\section{Experiment 2: influence of testosterone administration in spring}

In the controls (PHPn + oil; Fig. 4), the plasma testosterone concentration remained at basal levels over the experimental period (February-July). The administration of TP in April led to high plasma testosterone concentrations (range: 9-13 ng/ml) in March and April (Fig. 4), but did not affect scrotal circumference. Differences in horn growth rate (GLM repeated measures ANOVA, $P<0 \cdot 05)$ were seen in spring between the PHPn + TP and PHPn + oil groups. In both groups an increase $(t$-test for matched pairs, $P<0 \cdot 05)$ in horn growth occurred in April, but the monthly horn growth rate was greater (ANOVA, $P<0 \cdot 05$ ) in the $\mathrm{PHPn}+\mathrm{TP}$ group $(0 \cdot 8 \pm 0 \cdot 1 \mathrm{~cm}$ compared with $0.5 \pm 0 \cdot 1 \mathrm{~cm}$; Fig. 5$)$. Testosterone supplementation enhanced horn growth in June and September compared with controls (ANOVA, $P<0 \cdot 05$ ). No significant correlation $(P>0 \cdot 05)$ was seen between horn growth and plasma testosterone concentration during the horn elongation period in the PHPn + oil $\left(R^{2}=0 \cdot 01\right)$ and PHPn $+\mathrm{TP}\left(R^{2}=0 \cdot 06\right)$ groups. As the data suggest that TP treatment is associated with a delayed rather than an immediate stimulatory effect, retrospective correlation analysis was performed for plasma testosterone levels in March-April and horn growth 2 months later (May-June), and a significant correlation was detected in both the PHPn + oil $\left(R^{2}=0 \cdot 40, P=0 \cdot 06\right)$ and PHPn $+\mathrm{TP}\left(R^{2}=0 \cdot 37\right.$, $P<0 \cdot 05)$ groups. Treatment with TP had no effect on any of the studied sperm variables (Table 2).

\section{Discussion}

The effect of artificial photoperiod and anti-androgen or testosterone treatment on antler development in cervids has long been studied and in great detail (Wislocki et al. 1947, Goss 1968). However, to our knowledge, this is the first study on horn growth involving photoperiod and androgen manipulation in any bovid species. The present findings show that elevated levels of plasma testosterone concentrations are not required to stop horn growth during the mating season; neither do circulating testosterone levels need to fall to basal levels for the resurgence of horn growth to occur.

The possible effect of increasing testosterone levels arresting horn growth during the rutting season has been discussed for many years (Lincoln 1990, 1998, Santiago-Moreno et al. 2005a). More recent findings during a comparative study on two wild Caprinae species (mouflons and ibex; ToledanoDíaz et al. 2007) appear to support this hypothesis; in that work a delayed increase in plasma testosterone levels in the ibex species was accompanied by a delayed arrest of horn growth. However, anti-androgen treatment with CA did not prevent the physiological prevention of horn growth in November. This lack of effect contrasts with results obtained in red deer (Cervus elaphus) in which CA given during the hard antler phase caused antler casting followed by a period of intensive growth of new antlers (Jaczewski et al. 2004). Similar results have been reported for the Southern pudu (Pudu puda) when treated with CA (Bubenik et al. 2002). In addition, Bubenik (1982, 1990a) reported that treatment

Table 1 Sperm variables in ibexes belonging to the PHPn +oil $(n=4), \mathrm{PHPn}+\mathrm{CA}(n=4), \mathrm{PHPld}+\mathrm{CA}(n=4)$, and PHPld + oil $(n=4)$ groups

\begin{tabular}{|c|c|c|c|c|c|c|c|c|}
\hline & Vol $(\mu \mathrm{l})$ & $\begin{array}{l}\mathbf{C}\left(\times 10^{6}\right. \\
\text { sperm/ml })\end{array}$ & MOT (\%) & $\mathbf{Q M}(0-5)$ & NE $(\%)$ & NAR (\%) & HOST (\%) & MAB (\%) \\
\hline PHPn + oil & $261 \cdot 2$ & $1582 \cdot 2$ & $66 \cdot 7^{\mathrm{a}}$ & $2 \cdot 8$ & $73 \cdot 8$ & $81 \cdot 2$ & $56 \cdot 0$ & $29 \cdot 1^{b}$ \\
\hline $\mathrm{PHPn}+\mathrm{CA}$ & $258 \cdot 3$ & $715 \cdot 4$ & $70 \cdot 0^{\mathrm{a}}$ & $3 \cdot 1$ & $55 \cdot 0$ & $88 \cdot 6$ & $49 \cdot 3$ & $18 \cdot 8^{\mathrm{b}}$ \\
\hline $\mathrm{PHPld}+\mathrm{CA}$ & $365 \cdot 5$ & $1154 \cdot 8$ & $27 \cdot 8^{\mathrm{b}}$ & 1.9 & $67 \cdot 4$ & $83 \cdot 1$ & $57 \cdot 2$ & $61 \cdot 1^{\mathrm{a}}$ \\
\hline PHPId + oil & $244 \cdot 9$ & $1971 \cdot 1$ & $54 \cdot 0^{\mathrm{a}}$ & $2 \cdot 3$ & $69 \cdot 3$ & $83 \cdot 7$ & $67 \cdot 8$ & $41 \cdot 1^{\mathrm{a}}$ \\
\hline S.E.M. & $80 \cdot 4$ & $234 \cdot 7$ & $4 \cdot 7$ & $0 \cdot 2$ & $3 \cdot 8$ & $1 \cdot 9$ & $4 \cdot 7$ & $3 \cdot 9$ \\
\hline
\end{tabular}

Vol, ejaculate volume; C, sperm concentration; MOT, motile spermatozoa; QM, quality of motility; NE, sperm viability as determined by staining with nigrosineosin; HOST, plasma membrane integrity assessed by the hypo-osmotic swelling test; NAR, intact acrosomes (normal apical ridge); MAB, morphological abnormalities. Different letters within columns indicate significant differences $(P<0 \cdot 05)$. 


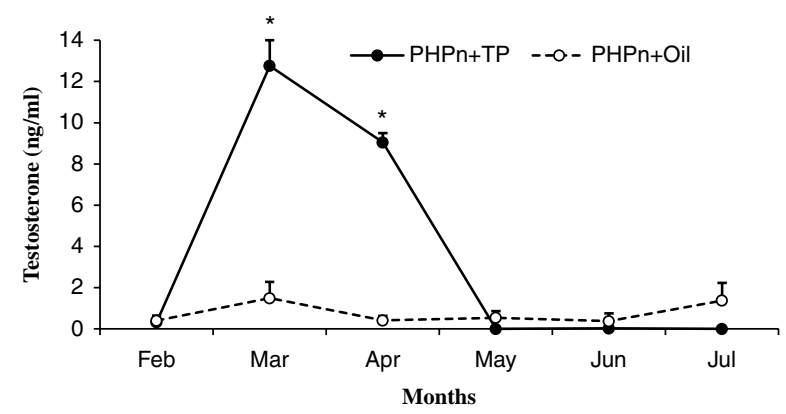

Figure 4 Plasma testosterone concentrations in control (PHPn + oil; open circle) and PHPn + TP (filled circle) animals. Asterisks indicate significant differences (ANOVA, $P<0 \cdot 05$ ) between groups within months. PHPn, natural photoperiodic conditions; TP, treated with testosterone propionate in olive oil; oil, olive oil without testosterone propionate.

with CA completely stopped antler growth in castrated white-tailed bucks (Odocoileus virginianus) and that antler regrowth stopped in two of six CA-treated castrated fallow deer bucks until CA treatment was ceased (Bartos et al. 2000). In other cervids, such as fallow deer (Dama dama), castration and CA treatment lead to premature antler casting (Goss 1990, Kierdorf et al. 1993), but CA treatment during the antler growth phase and main breeding season is reported not to affect the onset, duration or termination of antler growth (Kolle et al. 1993). This is inconsistent with Bubenik's suggestion that small circulating levels of androgen are required for antler growth (Bubenik 1990a).

Although the mechanism regulating antler growth has commonly been extrapolated to bovid horn growth (Lincoln 1998), the different histological characteristics of antler (compact bone) and horn (an epidermal structure covering an underlying bony core), and their different types of growth cycle (with periodic antler casting and regrowth in cervids), suggest substantial differences must exist in their mechanisms of control. The finding of the present first experiment that the timing of the horn growth cycle is not linked to seasonal variations in testosterone concentration was confirmed by the results of the second, in which the administration of TP coinciding with the natural increase in horn growth (Toledano-Díaz et al. 2007) did not prevent growth at all. Thus, testosterone does not act as an endocrine signal for the arrest of horn growth.

It might be argued that the inability of CA to prevent the autumnal reduction in horn growth despite its inducing basal testosterone levels suggests that even these low levels of this hormone are sufficient to block growth. However, this is unlikely because, in addition to the suppression of the release of hypothalamic GnRH by a negative feedback and subsequent androgen secretion (progestin action), CA blocks the androgen receptors (Neumann \& Töpert 1986, Neumann 1994).

The administration of testosterone in the spring did not affect the moment of horn growth resurgence, although it did allow for a higher rate of growth once it had begun.
Testosterone supplementation in April enhanced horn growth compared with controls, showing a stimulatory effect of testosterone on horn growth in the spring season. The stimulatory effect of TP was not observed until at least 2 months after its administration, suggesting a delayed effect rather an immediate effect. Under natural conditions, horn growth in wild bovids coincides with the springtime basal levels of testosterone; in fact it has been suggested that low concentrations of circulating androgens are required for horn growth (Lincoln 1998). In cervids, the size of, and bone mineralisation in, antlers is directly related to the testosterone concentration present before the rut; thus, antler characteristics are considered an indicator of change in testosterone secretion (Bubenik 1990b, 1991). Moreover, using castrated fallow deer as an experimental model, treated with or not with CA, it has been shown that the antlers of males with higher basal testosterone concentrations in the spring grow more rapidly (Bartos et al. 2000). In red deer stags, testosterone also appears to be responsible for the rate of antler growth in subadult and adult animals (Bartos et al. 2009). In bovids, this relationship between testosterone concentration and horn development has never been studied, but the present data strongly suggest that horn growth rate before the mating period is related to basal testosterone concentration. Indeed, horn growth has been likely related to spring testosterone levels in bighorn sheep (Ovis canadensis; Henderson \& Firebaugh 1997). Therefore, it would be expected that dominant males with greater horn development and sperm quality (Santiago-Moreno et al. 2007) show higher testosterone levels during the spring period of horn growth. Although Decristophoris et al. (2007) found no relationship between rutting season testosterone levels and dominance in Alpine ibex (Capra ibex), most studies in wild ruminants, such as Grant's gazelle (Nanger granti) and bighorn sheep, have found positive relationships between these variables (Pelletier et al. 2003, Ezenwa et al. 2012).

The persistence of horn growth during the autumn in the ibexes kept under long days clearly shows that such

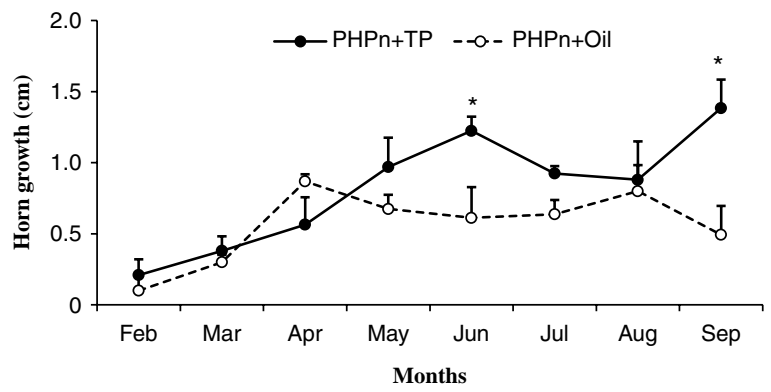

Figure 5 Horn growth in control (PHPn+oil; open circle) and PHPn + TP (filled circle) animals. Asterisks indicate significant differences (ANOVA, $P<0 \cdot 05$ ) between groups within months. $\mathrm{PHPn}$, natural photoperiodic conditions; TP, treated with testosterone propionate in olive oil; oil, olive oil without testosterone propionate. 
Table 2 Sperm variables in ibexes belonging to the PHPn+oil $(n=4)$ and PHPn+TP $(n=5)$ groups. No significant differences detected between groups

\begin{tabular}{|c|c|c|c|c|c|c|c|c|}
\hline & Vol $(\mu \mathrm{l})$ & $\begin{array}{l}\text { C }\left(\times 10^{6}\right. \\
\text { sperm/ml })\end{array}$ & MOT (\%) & $\mathbf{Q M}(0-5)$ & NE (\%) & NAR (\%) & HOST $(\%)$ & MAB (\%) \\
\hline PHPn+oil & $160 \cdot 7$ & $1344 \cdot 2$ & $79 \cdot 6$ & $3 \cdot 4$ & $59 \cdot 3$ & $66 \cdot 8$ & $55 \cdot 6$ & $37 \cdot 8$ \\
\hline $\mathrm{PHPn}+\mathrm{TP}$ & $201 \cdot 3$ & $818 \cdot 2$ & $63 \cdot 3$ & $2 \cdot 9$ & $59 \cdot 5$ & $53 \cdot 3$ & $53 \cdot 1$ & $42 \cdot 0$ \\
\hline S.E.M. & $221 \cdot 6$ & $234 \cdot 8$ & $7 \cdot 2$ & $0 \cdot 3$ & $5 \cdot 8$ & $6 \cdot 3$ & $5 \cdot 2$ & $6 \cdot 8$ \\
\hline
\end{tabular}

Vol, volume of the ejaculates; C, sperm concentration; MOT, motile spermatozoa; QM, quality of motility; NE, sperm viability by staining with nigrosin-eosin; HOST, plasma membrane integrity assessed by the hypo-osmotic swelling test; NAR, intact acrosomes (normal apical ridge); MAB, morphological abnormalities.

growth, at least during this period, is primarily modulated by photoperiodicity rather than any fluctuation in androgen levels. The lack of correlation between horn growth and plasma testosterone concentrations at this time supports the idea that this regulation is independent of testosterone secretion.

It is unclear how photoperiod may regulate horn growth, but recent studies suggest that prolactin, the plasma levels of which follow a trend roughly parallel to day length (Santiago-Moreno et al. 2003), may be involved. Certainly, the expression of prolactin receptors in epithelial cells in the horn growth area during spring is increased (Picazo et al. 2008). Steroid hormones, such as testosterone, are known to regulate populations of their own and other receptors (heterospecific regulation; Aragona et al. 1976), and prolactin receptors are known to fall in number under multi-hormonal regulation (Klemcke et al. 1990, Barash et al. 1992). Thus, testosterone might influence horn growth via the control of expression and/or binding activity of prolactin receptors in horn epithelial cells. The greater horn growth rate seen in ibexes with higher basal spring testosterone concentrations might be related to an increased expression of prolactin receptors.

The present results reveal a direct relationship between the rate of horn growth and the percentage of spermatozoa with morphological abnormalities. Artificial long days were the primary factor affecting the values recorded for the studied sperm variables. Certainly, the percentage of sperm abnormalities increased in ibexes maintained under this type of photoperiod. This agrees with the known inhibitory effect of long days on sexual activity in other short day breeders such as goats (for a review see Chemineau et al. (2008)). The sperm abnormalities reported for the $\mathrm{PHPn}+$ oil group in the second experiment (sperm samples recovered in spring) were similar to those seen in PHPld + CA and PHPld + oil groups (sperm samples recovered in autumn). This is clearly explained by the seasonal breeding activity in this species. The ibex has a short breeding season that runs from December to February (Santiago-Moreno et al. 2003). Semen quality improves in autumn, the number of sperm abnormalities being highest during spring and summer (Coloma et al. 2011). Hence, the present data support the idea that long days negatively affect sperm quality, with a high percentage of sperm abnormalities appearing, similar to the situation seen during the non-breeding season.

Although CA treatment maintained plasma testosterone concentrations at basal levels, under the natural photoperiod, CA treatment had no effect on testis size or sperm variables. This could be explained because the period of CA treatment was not very long. However, CA treatment and artificial long days (PHPld + CA group) produced a synergic inhibitory effect on scrotal circumference and sperm quality variables, significantly increasing the percentage of sperm abnormalities and reducing the percentage of motile spermatozoa. The most common morphological abnormalities in ibex sperm are defects in the tail (Coloma et al. 2011). Damage to the axonemal structure of the flagellum or its functioning (Gagnon \& de Lamirande 2006) during spermiogenesis may be caused by decreasing LH and FSH under long day or CA treatment. Testosterone, acting via its primary metabolites oestradiol and dihydrotestosterone, suppresses circulating $\mathrm{LH}$ concentrations, primarily by reducing the frequency of GnRH pulses (Hileman \& Jackson 1999). The administration of exogenous testosterone inhibits the pulsatile release of $\mathrm{LH}$ (Tilbrook et al. 1999) and can reversibly suppress spermatogenesis (Arsyad 1993). In humans it has been shown that longterm treatment with testosterone reduces testis size and reduces sperm concentration, motility, and viability, as well as affecting normal sperm morphology. But these effects are only observed about 10 weeks after the onset of treatment (Arsyad 1993). The fact that the TP treatment in the present work did not affect any of the studied sperm variables may suggest that the period of treatment (just 1 month) was too short for any to be noticed.

In conclusion, the timing of the horn growth cycle in the Iberian ibex is not linked to the seasonal variation in plasma testosterone concentration; rather, the photoperiod plays a major role in its regulation. The rate of horn growth before the mating season does, however, appear to be related to springtime plasma testosterone concentration, with higher basal levels leading to increased growth.

\section{Declaration of interest}

The authors declare that there is no conflict of interest that could be perceived as prejudicing the impartiality of the research reported.

Journal of Endocrinology (2012) 214, 155-163 


\section{Funding}

This work was supported by MICINN grants AGL2008-01747 and AGL2011-25495.

\section{Acknowledgements}

The authors thank the Environmental Council of Andalusia (Consejería de Medio Ambiente, Junta de Andalucía) for its unfailing help in the execution of the projects proposed, and the Sierra de Cazorla-Segura (Jaén) and Serranía de Ronda (Málaga) Game Reserves for the generous provision of animals. Special thanks are owed to the INIA animal husbandry team for help received in the handling and care of the experimental animals.

\section{References}

Aragona C, Bohnet HG \& Frisesen HG 1976 Induction of lactogen receptors II: studies on the liver of hypophysectomised male rats and on rats having a growth hormone secreting tumor. Endocrine Research Communications 3 199-208. (doi:10.3109/07435807609056900)

Arsyad KM 1993 Sperm function in Indonesian men treated with testosterone enanthate. International Journal of Andrology 16 355-361. (doi:10.1111/j. 1365-2605.1993.tb01362.x)

Barash I, Madar Z \& Gertler A 1992 Short-term in vivo regulation of prolactin receptors in the liver, testes, kidneys, and mammary gland of rats. Receptor 2 39-44.

Bartos L, Schams D, Kierdorf U, Fischer K, Bubenik GA, Siler J, Losos S, Tománek M \& Lastovkova J 2000 Cyproterone acetate reduced antler growth in surgically castrated fallow deer. Journal of Endocrinology 164 87-95. (doi:10.1677/joe.0.1640087)

Bartos L, Schams D \& Bubenik GA 2009 Testosterone, but not IGF-1, LH, prolactin or cortisol, may serve as antler-stimulating hormone in red deer stags (Cevus elaphus). Bone 44 691-698. (doi:10.1016/j.bone.2008.12.004)

Bubenik GA 1982 Endocrine regulation of the antler cycle. In Antler Development in Cervidae, pp 73-107. Ed. RD Brown. Kinsville, TX: Caesar Kleberg Wildl Res Inst.

Bubenik GA 1990a Neuroendocrine regulation of the antler cycle. In Horns, Pronghorns, and Antlers, pp 265-297. Eds GA Bubenik \& AB Bubenik. New York: Springer-Verlag.

Bubenik GA $1990 b$ The antler as a model in biochemical research. In Horns, Pronghorns, and Antlers, pp 474-487. Eds GA Bubenik \& AB Bubenik. New York: Springer-Verlag.

Bubenik GA 1991 Regulatory mechanisms of the antler cycle and the selection of deer breeding stock by endocrine tests. In Wildlife Production: Conservation and Sustainable Development, pp 521-529. Eds LA Renecker \& RJ Hudson. AFES Misc Publ No. 91-96, Fairbanks: University of Alaska Fairbanks.

Bubenik GA, Reyes E, Schams D, Lobos A, Bartos L \& Koerner F 2002 Effect of antiandrogen cyproterone acetate on the development of the antler cycle in Southern Pudu (Pudu puda). Journal of Experimental Zoology 292 393-401. (doi:10.1002/jez.10046)

Campbell RC, Dott HM \& Glover TD 1956 Nigrosin eosin as a stain for differentiating live and dead spermatozoa. Journal of Agricultural Science 48 1-8. (doi:10.1017/S002185960003029X)

Chemineau P, Guillaume D, Migaud M, Thiéry JC, Pellicer-Rubio MT \& Malpaux B 2008 Seasonality of reproduction in mammals: intimate regulatory mechanisms and practical implications. Reproduction in Domestic Animals 43 (Suppl 2) 40-47. (doi:10.1111/j.1439-0531.2008.01141.x)

Coloma MA, Toledano-Díaz A, Castaño C, Velázquez R, Gómez-Brunet A, López-Sebastián A \& Santiago-Moreno J 2011 Seasonal variation in reproductive physiological status in the Iberian ibex (Capra pyrenaica) and its relationship with sperm freezability. Theriogenology 76 1695-1705. (doi:10.1016/j.theriogenology.2011.07.001)

Courot M \& Ortavant R 1981 Endocrine control of spermatogenesis in the ram. Journal of Reproduction and Fertility. Supplement 30 47-60.
Decristophoris PMA, von Hardenberg A \& McElligott AG 2007 Testosterone is positively related to the output of nematode eggs in male Alpine ibex (Capra ibex) faeces. Evolutionary Ecology Research 9 1277-1292.

Ezenwa VO, Ekernas LS \& Creel S 2012 Unravelling complex associations between testosterone and parasite infection in the wild. Functional Ecology 26 123-133. (doi:10.1111/j.1365-2435.2011.01919.x)

Fandos P 1995 Factors affecting horn growth in male Spanish ibex (Capra pyrenaica). Mammalia 59 229-235. (doi:10.1515/mamm.1995.59.2.229)

Fowler ME 1993 Horns and antlers. In Zoo and Wild Animal Medicine: Current Therapy 3, pp 489-493. Ed. ME Fowler. Philadelphia, USA: WB Saunders Company.

Gagnon C \& de Lamirande E 2006 Controls of sperm motility. In The Sperm Cell. Production, Maturation, Fertilization, Regeneration, pp 108-133. Eds C de Jonge \& C Barratt. Cambridbe, UK: Cambridge University Press.

Geist V 1966 The evolutionary significance of mountain sheep horns. Evolution 20 558-566. (doi:10.2307/2406590)

Geist V 1991 On the taxonomy of giant sheep (Ovis ammon Linnaeus, 1766). Canadian Journal of Zoology 69 706-723. (doi:10.1139/z91-103)

Goss RJ 1968 Inhibition of growth and shedding of antlers by sex hormones. Nature 220 83-85. (doi:10.1038/220083a0)

Goss RJ 1990 Tumor-like growth of antlers in castrated fallow deer - an electron microscopic study. Scanning Microscopy 4 715-721.

Hafez ESE 1952 Studies on the breeding season and reproduction of the ewe. Journal of Agricultural Science 42 189-265. (doi:10.1017/ S0021859600056896)

Henderson RE \& Firebaugh JE 1997 Horn growth of a castrated Bighorn sheep, Ovis canadensis. The Canadian Field-Naturalist 111 475-477.

Hileman SM \& Jackson GL 1999 Regulation of gonadotrophin-releasing hormone secretion by testosterone in male sheep. Journal of Reproduction and Fertility. Supplement 54 231-242.

Jaczewski Z, Gizejewski Z \& Bartecki R 2004 The effect of cyproterone acetate on the antler cycle in red deer/Cervus elaphus L./. Reproductive Biology 4 165-176.

Jeyendran R, Van der ven H, Pérez-Peláez M, Crabo B \& Zaneveld L 1984 Development of an assay to assess the functional integrity of the human sperm membrane and its relation to other semen characteristics. Journal of Reproduction and Fertility 70 219-228. (doi:10.1530/jrf.0.0700219)

Kierdorf U, Schultz M \& Fischer K 1993 Effects of an antiandrogen treatment on the antler cycle of male fallow deer (Dama dama L.). Journal of Experimental Zoology 266 195-205. (doi:10.1002/jez.1402660305)

Klemcke HG, Amador AG \& Bartke A 1990 Hormonal regulation of testicular prolactin receptors and testosterone synthesis in Golden hamsters. Biology of Reproduction 43 162-168. (doi:10.1095/biolreprod43.1.162)

Kolle R, Kierdorf U \& Fischer K 1993 Effects of an antiandrogen treatment on morphological characters and physiological functions of male fallow deer (Dama dama L.). Journal of Experimental Zoology 267 288-298. (doi:10.1002/ jez.1402670307)

Lincoln G 1990 Correlation with changes in horns and pelage, but not reproduction, of seasonal cycles in the secretion of prolactin in rams of wild, feral and domesticated breeds of sheep. Journal of Reproduction and Fertility $\mathbf{9 0}$ 285-296. (doi:10.1530/jrf.0.0900285)

Lincoln G 1998 Reproductive seasonality and maturation throughout the complete life-cycle in the mouflon ram (Ovis musimon). Animal Reproduction Science 53 87-105. (doi:10.1016/S0378-4320(98)00129-8)

Neumann F 1994 The antiandrogen cyproterone acetate: discovery, chemistry, basic pharmacology, clinical use and tool in basic research. Experimental and Clinical Endocrinology 102 1-32. (doi:10.1055/s-0029-1211261)

Neumann F \& Töpert M 1986 Pharmacology of antiandrogens. Journal of Steroid Biochemistry 25 885-895. (doi:10.1016/0022-4731(86)90320-1)

Parsons P 1992 Fluctuating asymmetry: a biological monitor of environmental and genomic stress. Heredity 68 361-364. (doi:10.1038/hdy.1992.51)

Pelletier F, Barman J \& Festa-Bianchet M 2003 Faecal testosterona in bighorn sheep (Ovis canadensis): behavioural and endocrine correlates. Canadian Journal of Zoology 81 1678-1684. (doi:10.1139/z03-156)

Picazo A, López-Sebastian A, Toledano-Díaz A, Gomez-Brunet A \& Santiago-Moreno J 2008 Cellular localisation of prolactin receptors in horn growth area skin throughout the year in Spanish ibex (Capra pyrenaica hispanica). Reproduction in Domestic Animals 43 (Suppl 4) 79. 
Preston BT, Stevenson IR, Pemberton JM, Coltman DW \& Wilson K 2003 Overt and covert competition in a promiscuous mammal: the importance of weaponry and testes size to male reproductive success. Proceedings of the Royal Society of London. Series B, Biological Sciences 270 633-640. (doi:10.1098/rspb.2002.2268)

Pursel V \& Johnson L 1974 Glutaraldehyde fixation of boar spermatozoa for acrosome evaluation. Theriogenology 1 63-68. (doi:10.1016/0093691X(74)90008-9)

Roldan ERS, Cassinello J, Abaigar T \& Gomendio M 1998 Inbreeding, fluctuating asymmetry, and ejaculate quality in an endangered ungulate. Proceedings of the Royal Society of London. Series B, Biological Sciences 265 243-248. (doi:10.1098/rspb.1998.0288)

Santiago-Moreno J, Gómez-Brunet A, González-Bulnes A, Malpaux B, Chemineau P, Pulido A \& López-Sebastián A 2003 Seasonal ovulatory activity and plasma prolactin concentrations in Spanish ibex (Capra pyrenaica hispanica) maintained in captivity. Reproduction, Nutrition, Development 43 217-224. (doi:10.1051/rnd:2003023)

Santiago-Moreno J, Gómez-Brunet A, Toledano-Díaz A, González-Bulnes A \& López-Sebastián A 2005a Influence of age on the relationship between annual changes in horn growth rate and prolactin secretion in the European mouflon (Ovis gmelini musimon). Animal Reproduction Science 85 251-261. (doi:10.1016/j.anireprosci.2004.04.042)

Santiago-Moreno J, Gómez-Brunet A, González-Bulnes A, Toledano-Díaz A, Malpaux B \& López-Sebastián A $2005 b$ Differences in reproductive pattern between wild and domestic rams are not associated with inter-specific annual variations in plasma prolactin and melatonin concentrations. Domestic Animal Endocrinology 28 416-429. (doi:10.1016/j.domaniend. 2005.02.002)
Santiago-Moreno J, Toledano-Díaz A, Pulido-Pastor A, Gómez-Brunet A \& López-Sebastián A 2007 Horn quality and postmortem sperm parameters in Spanish ibex (Capra pyrenaica hispanica). Animal Reproduction Science 99 354-362. (doi:10.1016/j.anireprosci.2006.06.004)

Santiago-Moreno J, Coloma MA, Dorado J, Pulido-Pastor A,

Gómez-Guillamon F, Salas-Vega R, Gómez-Brunet A \& López-Sebastián A 2009 Cryopreservation of Spanish ibex (Capra pyrenaica) sperm obtained by electroejaculation outside the rutting season. Theriogenology 17 1253-1260. (doi:10.1016/j.theriogenology.2008.12.019)

Tilbrook AJ, Kretser DM \& Clarke IJ 1999 Seasonal changes in the negative feedback regulation of the secretion of the gonadotrophins by testosterone and inhibin in rams. Journal of Endocrinology 160 155-167. (doi:10.1677/joe. $0.1600155)$

Toledano-Díaz A, Santiago-Moreno J, Pulido-Pastor A \& López-Sebastian A 2007 Horn growth related to testosterone secretion in two wild Mediterranean ruminant species: the Spanish ibex (Capra pyrenaica hispanica) and European mouflon (Ovis orientalis musimon). Animal Reproduction Science 102 300-307. (doi:10.1016/j.anireprosci.2006.10.021)

Wislocki GB, Aub JC \& Waldo CM 1947 The effects of gonadectomy and the administration of testosterone propionate on the growth of antlers in male and female deer. Endocrinology 40 202-224. (doi:10.1210/endo-40-3-202)

Received in final form 3 May 2012

Accepted 29 May 2012

Made available online as an Accepted Preprint 29 May 2012 